\title{
INHALTSVERZEICHNIS
}

BLOCKDIAGRAMMTECHNIK

1. Grundlagen Der Blockdiagrammtechinik . . . . . . . . 15

1.1. Symbole . . . . . . . . . . . . . . 17

1.2. Einleitungsbeispiel . . . . . . . . . 19

1.3. Definition des Blockdiagramms ......... 21

1.4. Lineare und zyklische Blockdiagramme ...... 23

1.5. Verzweigungen .............. 25

1.6. Grobe und feine Blockdiagrame . . . . . . 27

1.7. Fehler in Blockdiagrammen . . . . . . . . 29

1.8. Prüfen der Blockdiagramme . . . . . . . . . 31

2. Aufbau Des Blockdiagramms ........... . 33

2.1. Allgemeine Hinweise . . . . . . . . . 35

2.2. Regeln für die Entwicklung der Blockdiagramme . . 37

2.3. Aufgabenstellung . . . . . . . . . . 37

2.4. Analyse der Aufgabenstellung ....... . 39

2.5. Kernstück der Aufgabe ............ 41

2.6. Abschliessen der Verarbeitung ........ 43

3. Optimierung Des Blockdiagramis ......... 45

3.1. Von der Vereinfachung zur komplexen Lösung . . 47

3.2. Schrittweise Ausbesserung des Blockdiagramms . 49

3.3. Programmierter Schalter ........... 49

3.4. Anwendung des Schalters ........... 51

3.5. Optimierte Lösung . . . . . . . . . . 53 
4. UeBUnGSTEIL .......................... 55

4.1. Einleitung zum Uebungsteil ........ 56

4.2. Aufgaben .............. 57

5. ANTWORTEN UND LÖSUNGEN . . . . . . . . . . . . 69

\section{INormierte Programierung}

1. Das Blockdiagramm Aus Der Sicht Der Normierung . . . . 73

2. Die Programmblöcke Der Normierten Programmierung . . . 77

3. Beispiel Monatsumsatz .............. 79

3.1. Der Vorlauf . . . . . . . . . 81

3.2. Die Dateieingabe ............ . 83

3.3. Die Dateiauswahl . . . . . . . . . . 83

3.4. Die Gruppenkontrolle . . . . . . . . . . 85

3.5. Die Gruppenverarbeitung . . . . . . . . 87

3.6. Die Einzelverarbeitung . . . . . . . . 89

3.7. Die Gruppensicherung . . . . . . . . . . . 91

3.8. Die Schlussverarbeitung . . . . . . . . 93

3.9. Die Programmablaufsteuerung . . . . . . . 95

4. Beispiel Jahresumsatz $1 \ldots . . . . . . .997$

4.1. Die Elemente der Dateisteuerung ...... 101

4.2. Die Dateiauswahl . . . . . . . . . 103

4.3. Die Steuerung der Eingabe-Dateien ...... 105

4.4. Die Gruppenverarbeitung im Beispiel Jahresumsatz 1... . . . . . . . . 107 
4.5. Die Einzelverarbeitung Methode A....... 109

4.6. Die Einzelverarbeitung Methode B ........ 111

5. Die Erweiterung Von Jahresumsatz 1 Zu Jahres-

UMSATZ $2 \ldots \ldots \ldots \ldots \ldots \ldots \ldots$

5.1. Die dateibezogenen Gruppenfelder DGR . . . . . . Il7

5.2. Das Lesegruppenfeld LGR . . . . . . . . . 119

5.3. Die dateineutralen Gruppenfelder NGR, AGR, SGR . . 121

5.4. Mehrstufige Gruppenkontrolle . . . . . . . 123

5.5. Selektives Lesen . . . . . . . . . . 125

5.6. Die Dateiprüfung DP ........... 127

6. Uebungsaufgabe . . . . . . . . . . . . . . . . . . . . . . .

6.1. Aufgabenstellung ............. 131

6.2. Lösung . . . . . . . . . . . . 134

7. AntwORTEN Und LöSUngen . . . . . . . . . . . . 145

ENTSCHEIDUNGSTABELLENTECHNIK . . . . . . . . . . . . . . 147

1. GRUNDLAGEN . . . . . . . . . . . . . . . . 147

1.1. Einführungsbeispiel . . . . . . . . . 149

1.2. Grundelemente von Entscheidungstabellen .....151

1.3. Begriffe der Entscheidungstabelle ...... 153

1.4. Zusammenfassung zu Kapitel I......... . 155

2. Arten Von Entscheidungstabellen . . . . . . . . . 157

2.1. Begrenzte Entscheidungstabellen . . . . . . 159

2.2. Erweiterte oder gemischte Entscheidungstabellen. . 161

2.3. Vollständige und unvollständige Entscheidungstabellen ............... . . 163 
2.4. Begrenzte, erweiterte und gemischte Standardtabellen . . . . ............ 165

3. Die Struktur Von Entscheidungstabellen . . . . . . 167

3.1. Redundanz ............... 169

3.2. Widerspruch (Kontradiktion) ........ 171

3.3. Ausschluss (Exklusion) und Einschluss

(Implikation) ............ . 173

3.4. Ueberschneidung (Intersection) ........ 175

3.5. Eindeutige und mehrdeutige Entscheidungstabellen . . . . . . . . . . . 177

3.6. Minimierung von ET . . . . . . . . . 179

3.7. Vollständigkeitskontrolle ........ . 181

3.8. Vollständigkeitskontrolle und Strukturanalyse mit Hilfe der Standardtabelle . . . . . 183

4. Anwendung In Analyse Und Programmierung $\ldots \ldots . . . .185$

4.1. Anwendung der ET in der Analyse . . . . . . 187

4.2. Anwendung der ET in der Programmierung . . . . 195

4.3. Anwendungsbeispiel ET-PAAREN ET-GRUPPEN . . . 199

4.4. Entscheidungstabellen-Vorübersetzer .....205

5. Vergleich Blockdiagramm-EntSCheidungstabelle . . . . . 207

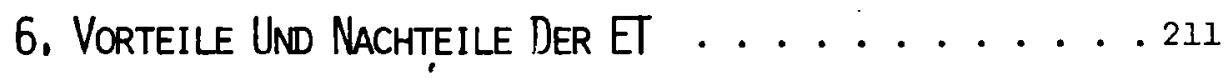

6.1. Vorteile................213

6.2. Nachteile ............. 215

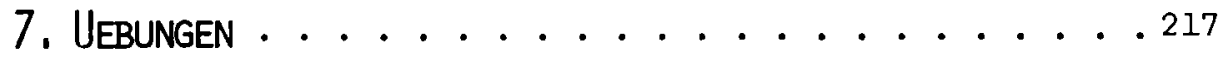

8. ANTWORTEN UND LÖSUNGEN . . . . . . . . . . . . 225 